\title{
Follicular dendritic cell sarcoma with extensive lymph node involvement: A case report
}

\author{
LI GU, CHUNHUI OUYANG and FANGGEN LU
}

\begin{abstract}
Division of Digestive Disease, Xiangya Second Hospital, Central South University, Changsha, Hunan 410011, P.R. China
\end{abstract}
Received August 3, 2014; Accepted April 17, 2015

DOI: $10.3892 / \mathrm{ol} .2015 .3234$

\begin{abstract}
Follicular dendritic cell sarcoma (FDCS) is an extremely rare neoplasm originating from the follicular dendritic cells in the lymphoid follicles. Current knowledge on its pathogenesis is limited and a well-defined treatment is required. The main channel through which clinicians obtain practical information on FDCS is via studying published case reports. To enrich the existing available literature and enhance the understanding of this rare disease, the present study describes a case of FDCS with extensive lymph node involvement in a 65 -year-old male patient whose predominant symptom was a recurrent fever. The diagnosis of FDCS was eventually established based on the immunohistochemical staining of biopsy specimens of the cervical lymph nodes. The patient improved and symptoms disappeared after one cycle of systemic chemotherapy.
\end{abstract}

\section{Introduction}

Follicular dendritic cells (FDCs), localized to primary and secondary B cell follicles, are unique immune cells participating in the regulation of humoral immune responses. The cells facilitate the activation of B cells, as they trap and retain antigens in the form of highly immunogenic immune complexes (1). A tumor arising from these FDCs is termed a FDC sarcoma (FDCS), and since Monda et al (2) first described FDCS in 1986, 200 cases have been reported in the English literature. In the majority of cases, FDCS presents as a painless, slow-growing well-circumscribed mass, with no constitutional symptoms, such as fever, night sweats, and weight loss (3). The diagnosis of FDCS depends on an array of morphological, histological, electron microscopic and, most importantly, immunohistochemical studies. Surgical resection

Correspondence to: Professor Fanggen Lu, Division of Digestive Disease, Xiangya Second Hospital, Central South University, 139 Middle Renmin Road, Changsha, Hunan 410011, P.R. China E-mail: lufanggen_xy2yy@163.com

Key words: follicular dendritic cell sarcoma, follicular dendritic cell tumor, case report remains the cornerstone of treatment. The efficacy of adjuvant therapy (chemotherapy or radiation) is yet unclear $(4,5)$. Cervical and intraabdominal lymph nodes are the most frequently affected nodal sites. In addition, various extranodal sites can also be involved, particularly in the liver, lungs and tonsils (6). The current study presents the second published case of FDCS with extensive lymph node involvement, as to the best of our knowledge, only one case has been reported previously (7). Written informed consent was obtained from the patient's family for the publication of this case report.

\section{Case report}

Patient presentation. A 65-year-old male presented to the Xiangya Second Hospital (Central South University, Changsha, Hunan, China) in July 2013 with a recurrent fever, abdominal distension and mild edema of the lower limbs that had persisted for 2 weeks. There was no significant past medical history. The physical examination revealed rebound tenderness in the abdomen, a palpable enlarged liver and shifting dullness. The laboratory test results of note were as follows: A white blood cell count of $6,200 / \mu 1$ (normal, 4000-10000/ $\mu 1$ ), a blood neutrophil percentage of 78.30 (normal, 50-70\%), an erythrocyte sedimentation rate of $40 \mathrm{~mm} / \mathrm{h}$ (normal, $<20 \mathrm{~mm} / \mathrm{h}$ ), a C-reactive protein level of $96.80 \mathrm{mg} / \mathrm{l}$ (normal, $<10 \mathrm{mg} / \mathrm{l}$ ) and a procalcitonin level of $0.25 \mathrm{ng} / \mathrm{ml}$ (normal, $<0.5 \mathrm{ng} / \mathrm{ml}$ ). Mycotic spores were found in a stool sample. Routine biochemical analysis revealed a marked increase in $\gamma$-glutamyl transpeptidase and alkaline phosphatase levels 134.2 U/1 (normal, 9.0-39.0 U/1) and 217.4 U/1 (normal, 42.0-141.0 U/1) respectively. The patient underwent an ultrasound of the abdomen, which revealed multiple gallbladder stones, cholecystitis, enlargement of the liver and spleen, multiple cysts on the kidneys and small amounts of ascites. The first clinical impression that was formed to account for the ascites and recurrent fever was one of infection, and empirical clinical treatment using the antibiotic moxifloxacin (400 mg/day for 7 days) combined with diuretic treatment [frusemide $(20 \mathrm{mg} /$ day $)$ and aldactone, (60 mg/day) for 10 days] was initiated prior to the outcome of a bacterial culture. Meanwhile, the patient received a computed tomography (CT) scan to further identify possible reasons for the recurrent fever, as well as the enlargement of the liver and spleen. The CT scan detected extensive enlargement of the lymph nodes in the mediastinal, retroperitoneal 

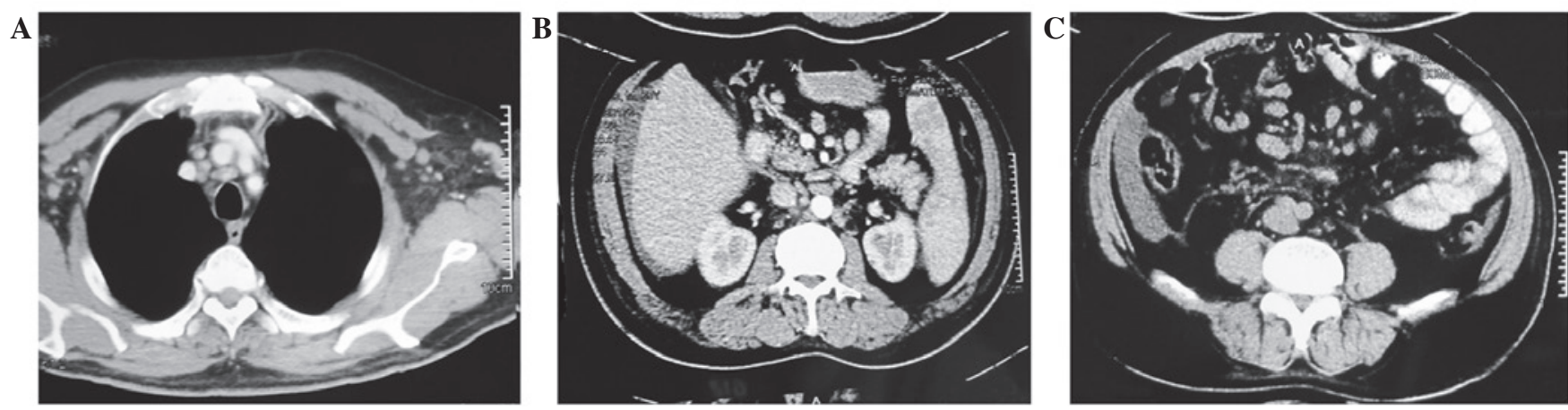

Figure 1. Computed tomography showing extensive involvement of enlarged lymph nodes in the (A) mediastinal, (B) retroperitoneal and (C) mesenteric areas.
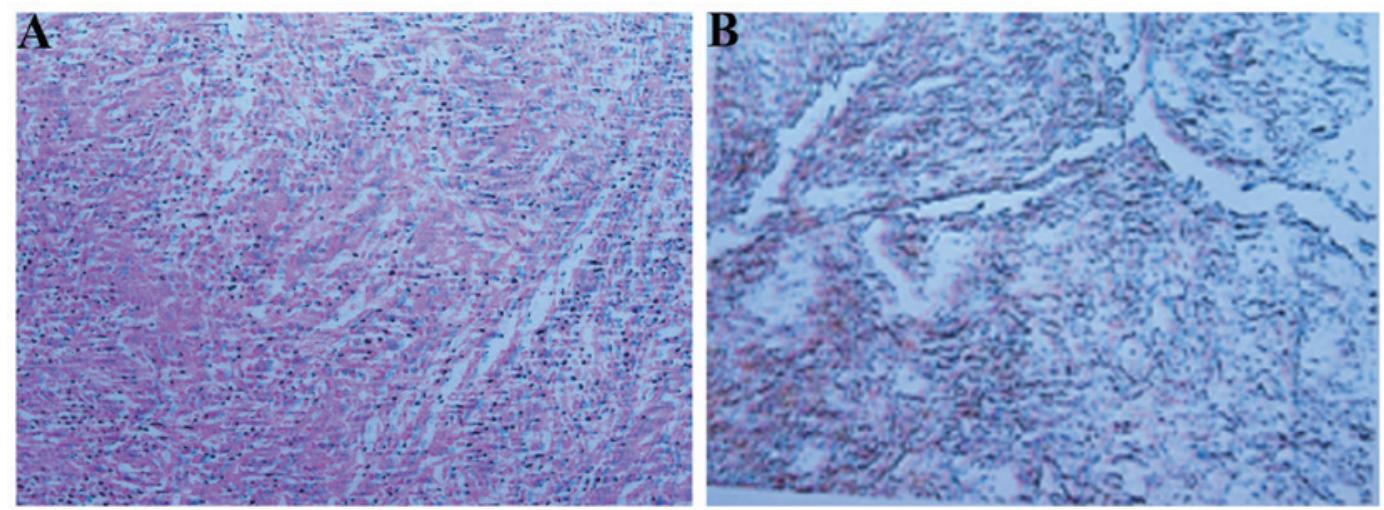

Figure 2. (A) Perivascular proliferation of spindle cells. (B) Immunohistochemical diffuse cytoplasmic positivity for cluster of differentiation 23 . Hematoxylin and eosin staining; magnification, $x 100$.

and mesenteric areas (Fig. 1). Color Doppler ultrasonography of the neck revealed multiple enlarged cervical lymph nodes, while a bone marrow specimen showed no malignancy. An excisional biopsy of a cervical lymph node was immediately performed, which revealed the existence of a poorly-differentiated malignant tumor.

Histological findings. Macroscopically, the cervical lymph node was $1 \times 0.3 \times 0.8 \mathrm{~cm}$ in size. On immunohistochemical (IHC) staining, the diagnostic antibodies used included antibodies against cluster of differentiation (CD) 23(++) (Fig. 2), CD21(++), S-100(-), D1a(-), CD3(+), CD31(-), CD45RO(+), $\mathrm{CK}(-), \mathrm{CD} 79 \mathrm{a}(+)$ and CD20(+). The intensity of the dye color was graded as 0 (no color), 1 (light yellow), 2 (light brown) or 3 (brown), and the number of positive cells was graded as $0(<5 \%), 1(5-25 \%), 2(25-50 \%), 3(51-75 \%)$ or $4(>75 \%)$. The two grades were added together and specimens were assigned one of four staining levels based on this score: 0-1 (-), $2(+)$, $3-4(++)$ and $>5(+++)$. Given that FDCS is specifically immunopositive to $\mathrm{CD} 21, \mathrm{CD} 35$, and/or CD23, a diagnosis of FDCS was determined based on the immunohistochemical staining result.

Treatment and outcome. Following one cycle of cyclophosphamide [0.1 g intravenously, day 1], epirubicin $(110 \mathrm{mg}$ intravenous infusion, day 1), vincristine (2 mg intravenously, day 1) and prednisone (100 mg orally, days 1-5) (CHOP) chemotherapy, the patient improved and symptoms of recurrent fever and abdominal distension disappeared. The patient will continue to receive consolidation chemotherapy (a further cycle of $\mathrm{CHOP}$ ) and undergo regular follow-up examinations.

\section{Discussion}

FDCS is a rare low- to intermediate-grade malignant tumor arising from germinal centers. The etiology and pathogenesis of FDCS are unclear. Among the reported cases of patients with FDCS, a small subset of cases are believed to be associated with Epstein-Barr virus, with the majority of these cases involving the liver or spleen and presenting with an inflammatory pseudotumor-like morphology $(8,9)$. Additionally, patients with a background of hyaline-vascular Castleman disease have been reported be at greater risk of FDCS, although the exact association between the conditions remains unknown $(10,11)$.

As a whole, FDCS involves the lymph nodes in one-half to two-thirds of cases, with the cervical nodes being the most common site (6). In the present case, the lymph nodes in the neck and the mediastinal, retroperitoneal and mesenteric areas were extensively affected. In past decades, a wide variety of extranodal sites have been reported, including the tonsils, liver, spleen, oral cavity, gastrointestinal tract, bones, soft tissues, skin and breasts (6).

The diagnosis of FDCS remains challenging, and a definitive diagnosis of this uncommon tumor depends on the distinctive histological morphology and IHC profile. The 
traditional diagnostic markers of FDCS mainly include CD21, CD23, CD35 and clusterin. However, it should be noted that the markers of FDC are not routinely used in IHC studies.

Among the published cases, surgery was used as the mainstay of treatment for early FDCS (3). Adjuvant chemotherapy or radiotherapy were reported to be used for the late disease management in several cases, with therapies consisting of the CHOP regimen, the ifosfamide, carboplatin and etoposide regimen, and the Adriamycin, bleomycin, vincristine and dacarbazine regimen (12).

In the present case, the fundamental cause of the enlargement of the liver and spleen remains in question. It is uncertain whether the enlargement of the liver and spleen was associated with the FDCS. Choi et al (7) described a case of FDCS with extensive lymph nodes being affected. In that case, the CT scan also revealed mild splenomegaly. However, there was no mention of the turnover of the mild splenomegaly following two cycles of $\mathrm{CHOP}$, therefore a conclusion cannot be drawn from this data. As a result, detailed follow-up records will be maintained in the present case.

Follicular dendritic cell sarcoma is extremely rare. From a diagnostic perspective, the pathologist should increase awareness regarding these tumors and further develop means for their molecular characterization. From a therapeutic perspective, although surgery is the primary treatment, when feasible, a multimodal approach and personalized treatment should be considered. Due to the rarity of this tumor, its optimal treatment yet to be defined, and multicenter cooperation and enrollment of patients in well-designed clinical studies are necessary to establish this.

\section{References}

1. Park CS and Choi YS: How do follicular dendritic cells interact intimately with B cells in the germinal centre. Immunology 114: 2-10, 2005.

2. Monda L, Warnke R and Rosai J: A primary lymph node malignancy with features suggestive of dendritic reticulum cell differentiation. A report of 4 cases. Am J Pathol 122: $562-572,1986$.

3. Dalia S, Shao H, Sagatys E, et al: Dendritic cell and histiocytic neoplasms: Biology, diagnosis, and treatment. Cancer Control 21: 290-300, 2014

4. Karligkiotis A, Contis D, Bella M, et al: Pediatric follicular dendritic cell sarcoma of the head and neck: A case report and review of the literature. Int J Pediatr Otorhinolaryngol 77: 1059-1064, 2013

5. Hu T, Wang X, Yu C, et al: Follicular dendritic cell sarcoma of the pharyngeal region. Oncol Lett 5: 1467-1476, 2013.

6. Saygin C, Uzunaslan D, Ozguroglu M, Senocak M and Tuzuner N: Dendritic cell sarcoma: A pooled analysis including 462 cases with presentation of our case series. Crit Rev Oncol Hematol 88: 253-271, 2013.

7. Choi BS, Baek JH, Shin YM, et al: Follicular dendritic cell sarcoma: A case report and review of the literature. Cancer Res Treat 42: 121-124, 2010

8. Shek TW, Ho FC, Ng IO, Chan AC, Ma L and Srivastava G: Follicular dendritic cell tumor of the liver. Evidence for an Epstein-Barr virus-related clonal proliferation of follicular dendritic cells. Am J Surg Pathol 20: 313-324, 1996.

9. Arber DA, Kamel OW, van de Rijn M, et al: Frequent presence of the Epstein-Barr virus in inflammatory pseudotumor. Hum Pathol 26: 1093-1098, 1995.

10. Meijs M, Mekkes J, van Noesel C, et al: Paraneoplastic pemphigus associated with follicular dendritic cell sarcoma without Castleman's disease; treatment with rituximab. Int J Dermatol 47: 632-634, 2008.

11. Yamamoto K, Yoshida M, Yamamoto M, et al: An abdominal follicular dendritic cell tumor in Castleman's disease. Rinsho Ketsueki 45: 1033-1038 (In Japanese).

12. Kairouz S, Hashash J, Kabbara W, et al: Dendritic cell neoplasms: An overview. Am J Hematol 82: 924-928, 2007. 